\title{
The Role of Fear of Negative Evaluation in Predicting Depression and Quality of Life Four Years after Bariatric Surgery in Women
}

\author{
Claire Elizabeth Adams ${ }^{1}$, Valerie Harwell Myers ${ }^{2}$, Brooke Louise Barbera ${ }^{1}$, \\ Phillip Jerome Brantley ${ }^{2}$ \\ ${ }^{1}$ Louisiana State University, Baton Rouge, USA; \\ ${ }^{2}$ Pennington Biomedical Research Center, Baton Rouge, USA. \\ Email: cadams44@gmail.com \\ Received February $7^{\text {th }}, 2011$; revised April $15^{\text {th }}, 2011$; accepted May $17^{\text {th }}, 2011$.
}

\begin{abstract}
The purpose of this study was to investigate the role of fear of negative evaluation (FNE) in predicting psychosocial functioning (depression and quality of life (QOL)) as a function of amount of weight lost four years after weight loss surgery among women. Four years after gastric bypass surgery, female participants $(\mathrm{N}=29)$ completed measures of FNE, depression, and QOL via telephone. Height and weight (pre- and post-surgery) were obtained from medical charts and current primary care physicians. FNE was not related to depression or QOL among patients who lost less weight. However, FNE predicted greater depression and worse QOL among those who had lost more weight four years after surgery. Several potential explanations are explored. Regardless of why social concerns are related to mood and QOL for certain individuals post-surgery, psychosocial therapy that addresses life transitions after weight loss, with particular focus on concerns about interpersonal evaluation, might improve mental health outcomes for women after bariatric surgery.
\end{abstract}

Keywords: Bariatric Surgery, Obesity, Fear of Negative Evaluation, Social Anxiety, Quality of Life

\section{Introduction}

Candidates for bariatric surgery often indicate feeling stigmatized and having lower quality of life (QOL) due to their weight (Sarwer, Fabricatore, Eisenberg, Sywulak, \& Wadden, 2008). Obese individuals often experience high levels of interpersonal sensitivity, social anxiety, and depression (Grilo, Wilfley, Jones, Brownell, \& Rodin, 1994; Petroni et al., 2007). Fear of negative evaluation (FNE; Leary, 1983; Watson \& Friend, 1969), or concern about the prospect of being negatively evaluated by others, may contribute to a great deal of psychological distress and lower QOL for certain obese individuals.

Much research suggests that dramatic weight loss can improve mood, quality of life, and body image, and greater weight loss is often associated with better psychological outcomes (Foster, Wadden, \& Vogt, 1997; Guisado, Vaz, Alarcón, López-Ibor, Rubio, \& Gaite, 2002; Herpertz, Kielmann, Wolf, Langkafel, Senf, \& Hedebrand, 2003; Karlsson, Taft, Rydén, Sjöström, \& Sullivan, 2007). However, some bariatric surgery patients indicate significant symptoms of depression and body dissatisfaction post-surgery (Sarwer et al., 2008). Furthermore, patients who do experience mental health benefits often report a peak in improvements after initial weight loss, with mental health indices and health-related QOL diminishing over subsequent years (Karlsson et al., 2007; Mathus-Vliegen, 2007; Waters, Pories, Swanson, Meelheim, Flickinger, \& May, 1991). There is a need for more research utilizing specific measures of psychological functioning rather than relying on global measures of mental health, as well as assessing long-term psychological outcomes, as initial gains may dissipate with time (Bocchieri, Meana, \& Fisher, 2002). Research should also investigate which patients are particularly prone to psychological distress post-surgery and why.

FNE is defined as the extent to which people are concerned about the prospect of negative interpersonal evaluation. Individuals with high levels of FNE tend to experience more anxiety in social situations and attempt to avoid interpersonal encounters that they perceive to be threatening (Leary, 1983). A great deal of research has described associations between FNE, body dissatisfaction, and cognitions and behaviors related to disordered eating among underweight and normal-weight populations. For example, social anxiety is often comorbid with eating disorders, and McClintock and Evans (2001) found a direct link between FNE and disordered eating. Similarly, Gilbert and Meyer (2005) reported that FNE longitudinally predicted increased bulimic attitudes over a 7-month period. In addition, Lundgren, Anderson, and Thompson (2004) reported that fear of negative appearance evaluation predicted body image dissatisfaction and dysfunctional eating attitudes. Taken together, these findings suggest that FNE may contribute to body dissatisfaction, dysfunctional eating, and associated psychological distress and decreased QOL among individuals concerned about their weight. FNE may be one important factor contributing to psychological distress and decreased QOL for patients after bariatric surgery. However, there is a dearth of information on FNE in participants seeking weight loss treatment.

Although research has documented psychosocial distress and social anxiety among individuals with obesity and eating disorders, specific relationships between FNE and QOL in obese populations (and those receiving bariatric surgery) have not been examined. The role of FNE post-bariatric surgery, as well as its relation to depression and QOL after weight loss maintenance, is unclear. For example, FNE and depression are typi- 
cally positively correlated (Duke, Krishnan, Faith, \& Storch, 2006); however, relationships between FNE, depression, and QOL in patients who lose more versus less weight are unknown. The purpose of this study was to investigate the role of FNE in psychosocial functioning after weight loss surgery.

The Office of Group Benefits (OGB), Louisiana's managed medical insurance program for state employees and their dependents, initiated a study in 2003 with the Louisiana State University School of Medicine to investigate outcomes of bariatric surgery. OGB teamed with Pennington Biomedical Research Center for a follow-up study to examine outcomes four years after surgery. Principal medical and psychosocial outcomes are described elsewhere (Myers, Adams, Barbera, \& Brantley, in press). The present study is a sub-analysis of this follow-up study.

\section{Method}

\section{Participants}

Participants were obese individuals (body mass index (BMI) $>40$ and $<60 \mathrm{~kg} / \mathrm{m}^{2}$ ) between the ages of 35 and 60 who denied comorbid malignancy or heart disease.

\section{Measures}

Weight. Pre-surgical weight and most current weight available were obtained from medical charts.

Short Form (36) Health Survey (SF-36; Ware, 1993). The SF-36 is a self-report health profile that includes eight subscales indicating QOL in several domains (physical function, role function-physical, role function-emotional, bodily pain, general health, social function, psychological well-being/mental health, and vitality). Two summary scales, the Physical Component Summary Score (PCS) and Mental Component Summary Score (MCS), are calculated. The SF-36 is widely used and psychometrically sound. In the current study, there was good evidence for reliability and validity of its administration by telephone; internal consistency was excellent (Cronbach's alpha $=0.97$ for the entire scale; ranged $.83-.96$ for subscales), and lower SF-36 scores were associated with higher levels of depressive symptoms.

Beck Depression Inventory-II (BDI-II; Beck, Steer, Ball, \& Ranieri, 1996). The BDI-II, a 21-item self-report scale, is a widely-used measure of depressive symptoms with good psychometric properties. Reliability of the telephone-administered BDI-II in the current study was excellent (Cronbach's alpha $=.98)$.

Brief Fear of Negative Evaluation Scale (BFNE; Leary, 1983). The BFNE was created as a shortened form of Watson and Friend's (1969) 30-item FNE, designed to assess degree of concern about the prospect of negative interpersonal evaluation. Leary's (1983) 12-item version of the scale showed good internal consistency and correlated highly with the original scale. However, more recently researchers (e.g., Duke et al., 2006; Rodebaugh, Woods, Thissen, Heimberg, Chambless, \& Rapee, 2004) have suggested the use of only the eight straightforwardly-worded items on the BFNE, as these items comprise a single factor with excellent internal consistency and correlate as expected with measures of loneliness and depression. Thus, only these eight items were included in the present study.

\section{Procedure}

In 2003, OGB informed 189, 398 insured members about a study on gastric bypass surgery. All morbidly obese (BMI > 40) adult members who wished to be considered for the surgery were mailed packets including details of the study, a consent form and HIPAA waiver. Those OGB members who returned the documentation $(\mathrm{N}=911)$ were mailed an additional letter requesting a scheduled telephone interview. Additional screening processes included self-report anthropometric and health information, and clinic visits in which standard diagnostic and preoperative testing was conducted. To be eligible for the surgery, participants needed to be between the ages of 35 and 60 , have a BMI greater than 40 but less than 60 , and not be diagnosed with heart disease or malignancy. A total of 40 OGB members were selected for the surgery. The surgeries (which were all laparoscopic Roux-en-Y gastric bypass procedures) occurred between April and November 2004. One postsurgical death occurred.

In 2008, data collection was conducted via telephone and chart review. Thirty of 39 patients completed psychosocial measures via telephone; the remaining nine were unable to be reached by telephone. Only one of the patients who completed psychosocial measures was male; thus, the sample was restricted to the 29 women who completed measures. Height and weight (pre- and post-surgery) were obtained from medical charts and current primary care physicians. All aspects of data collection and management were reviewed and approved by the Pennington Biomedical Research Center Institutional Review Board.

\section{Results}

\section{Demographics}

Participants were predominantly Caucasian (22 Caucasians, 6 African Americans, 1 Hispanic). The average age was 51.9 ( $\mathrm{SD}=7.60$ years). The sample was highly educated, with $75 \%$ attending at least some college. Weight loss and psychological outcomes are presented by Myers et al. (in press). Participants in the current sample lost an average of $95.43(\mathrm{SD}=22.40)$ pounds (range $63-170 \mathrm{lbs}$.), or a loss of $33.62 \%$ of initial weight $(\mathrm{SD}=6.68 \%$, ranging $21 \%-49 \%$ ). Average pre-surgical BMI was 47.95 ( $\mathrm{SD}=4.28$; range 41.2 - 58.2). Average BMI 4-years post-surgery was $31.82(\mathrm{SD}=4.59$; range 24.21 43.18).

Participants' mean BFNE total score was $14.10(\mathrm{SD}=6.62)$, suggesting a generally low degree of concern about interpersonal evaluation. Myers et al. (in press) reported that on the BDI-II, the total sample reported minimal depressive symptoms, and that SF-36 scores suggested good/normal levels of general mental and physical QOL. Descriptive statistics for demographic variables, percent weight loss, and psychosocial measures are shown in Table 1. In addition, this information is given for participants who lost relatively more versus less weight, as defined by a median split (median percent weight lost $=.33$ ). Independent samples t-tests and chi-square analyses indicated that participants who lost more versus less weight did not differ 
significantly on demographic variables, baseline weight, FNE, depression, or any type of QOL, $p \mathrm{~s}>.14$.

\section{Relationships between FNE, Weight Loss, and Psychosocial Outcomes}

First, Pearson product moment correlations were conducted between FNE, BDI-II, mental and physical QOL scores from the SF-36, and percent of weight loss. These correlations are presented in Table 2. FNE was correlated with higher levels of depressive symptoms. However, FNE was not related to physical or mental QOL, or percent of weight lost after surgery.

Next, a series of hierarchical regression analyses were conducted predicting QOL and BDI-II from FNE, percentage of weight lost, and the interaction between FNE and weight loss, controlling for age, race, and education. Data were examined for adherence to assumptions of normality and homoscedasticity, and outliers greater than 3.3 standard deviations from their predicted mean were removed (as suggested by Tabachnick \& Fidell, 2007).

There were no significant main effects of demographic variables, FNE, or percent weight loss on depression or QOL, ps $>.05$. However, results revealed significant interactions between FNE and weight loss in predicting mental QOL $(F(1,17)$ $\left.=7.21, p=.01, s r^{2}=.24\right)$, and depression $(F(1,19)=10.92, p$ $\left.=.004, s r^{2}=.30\right)$. In addition, there was a marginally significant interaction in predicting physical QOL $(F(1,17)=3.08, p=.09$, $\left.s r^{2}=.14\right)$.

Interactions were decomposed with analyses of simple slopes at specified levels of weight loss as recommended by Aiken and West (1991). In predicting mental QOL, FNE was not related to MCS scores among participants who lost less weight (i.e., those $1 \mathrm{SD}$ below the mean of percent weight loss), $p=.56$. However, among participants who lost more weight (i.e. $1 \mathrm{SD}$ above the mean), FNE predicted worse mental QOL, $t(21)=3.67, p=$ $0.001, s r^{2}=.38$. See Figure 1 . The same pattern emerged for physical QOL. FNE was not related to PCS scores among participants who lost less weight, $p=.21$; however, among those who lost more weight, FNE predicted worse physical QOL, $t(21)=2.13, p=.04, s r^{2}=.17$. Similarly, among participants who lost less weight, FNE was not related to depression, $p$ $=.48$. However, among participants who lost more weight, FNE predicted higher depression, $t(23)=4.42, p<.001, s r^{2}$ $=.45$.

In examining specific aspects of QOL, significant interactions between FNE and weight loss emerged for physical function $\left(F(1,17)=5.07, p=.04, s r^{2}=.19\right)$, general health $(F(1,17)$ $\left.=6.47, p=.02, s r^{2}=.22\right)$, social function $(F(1,17)=9.69, p$ $\left.=.006, s r^{2}=.30\right)$, and mental health $(F(1,17)=11.46, p=.004$, $\left.s r^{2}=.31\right)$. Post-hoc analyses of simple slopes for each subscale were consistent with results for PCS and MCS indices; FNE was not related to QOL among participants who lost less weight; however, among those who lost more weight, FNE predicted worse QOL related to physical function, general health, social function, and mental health. Table 3 shows correlations between FNE and domains of QOL for participants who lost more versus less weight based on a median split (median percent weight lost $=.33$ ).

Table 1.

Demographic Characteristics, Percent Weight Loss, and Psychosocial Variables for the Total Sample and Split by Percent Weight Loss (standard deviations in parentheses).

\begin{tabular}{lccc}
\hline & Total Sample $(n=29)$ & Less Weight Loss $(21 \%-32 \% ; n=12)$ & More Weight Loss $(34 \%-49 \% ; n=15)$ \\
\hline Age & $51.90(7.60)$ & $54.25(3.86)$ & $48.73(8.66)$ \\
\% Caucasian & $75.9 \%$ & $83.3 \%$ & $73.3 \%$ \\
\% Attending at Least Some College & $75.0 \%$ & $75.0 \%$ & $73.3 \%$ \\
Baseline BMI & $47.95(4.28)$ & $48.13(4.37)$ & $47.71(4.63)$ \\
\% Weight Loss 4 Years after Surgery & $33.62 \%(6.68 \%)$ & $27.99 \%(3.72 \%)$ & $38.12(4.81)$ \\
FNE & $14.10(6.62)$ & $15.58(8.16)$ & $13.20(5.44)$ \\
BDI-II & $5.69(11.50)$ & $4.67(8.66)$ & $6.20(14.02)$ \\
PCS & $49.96(10.35)$ & $50.80(10.92)$ & $51.51(8.86)$ \\
MCS & $52.77(10.10)$ & $53.43(8.31)$ & $53.36(11.41)$ \\
\hline
\end{tabular}

BMI = Body Mass Index; FNE = Brief Fear of Negative Evaluation Scale; BDI-II = Beck Depression Inventory, $2^{\text {nd }}$ Edition; PCS $=$ Physical Component Summary Score of Short-Form Health Survey (SF-36); MCS = Mental Component Summary Score of SF-36.

Table 2.

Correlations between Fear of Negative Evaluation, Depression, Quality of Life, and Weight Loss.

\begin{tabular}{lccccc}
\hline & FNE & BDI-II & PCS & MCS & \% Weight Loss \\
\hline FNE & 1 & $.36^{*}$ & $-.17 \mathrm{~ns}$ & $-.32 \mathrm{~ns}$ & $-.08 \mathrm{~ns}$ \\
BDI-II & $.36^{*}$ & 1 & $.77^{* *}$ & $.76^{* *}$ & $.07 \mathrm{~ns}$ \\
PCS & $-.17 \mathrm{~ns}$ & $.77^{* *}$ & 1 & $.50^{* *}$ & $.14 \mathrm{~ns}$ \\
MCS & $-.32 \mathrm{~ns}$ & $.76^{* *}$ & $.50^{* *}$ & 1 & $-.12 \mathrm{~ns}$ \\
$\%$ Weight Loss & $-.08 \mathrm{~ns}$ & $.07 \mathrm{~ns}$ & $.14 \mathrm{~ns}$ & $-.12 \mathrm{~ns}$ & 1 \\
\hline
\end{tabular}

FNE = Brief Fear of Negative Evaluation Scale; BDI-II = Beck Depression Inventory, 2nd Edition; PCS = Physical Component Summary Score of Short-Form Health Survey (SF-36); MCS = Mental Component Summary Score of SF-36. $* p<.05 ; * * p<.01$. 


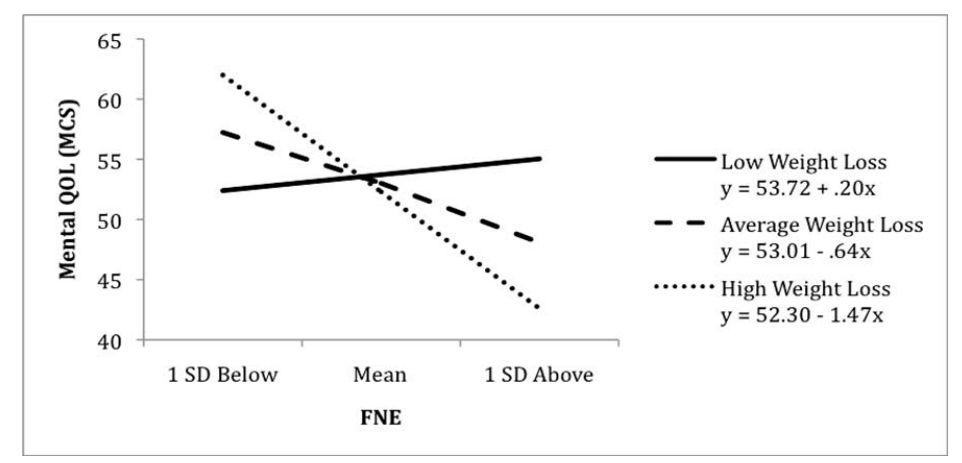

Figure 1.

Interaction of Fear of Negative Evaluation (FNE) by percent weight loss for Mental Quality of Life (QOL).

Table 3.

Correlations between Fear of Negative Evaluation, Depression, and Quality of Life Subscales among Participants who Lost More versus Less Weight.

\begin{tabular}{lcc}
\hline & $\begin{array}{c}\text { Less Weight Loss }(21 \%-32 \%) \\
(n=12)\end{array}$ & $\begin{array}{c}\text { More Weight Loss }(34 \%-49 \%) \\
(n=15)\end{array}$ \\
\hline BDI-II & $.02^{\mathrm{ns}}$ & $.68^{* *}$ \\
Physical Functioning & $.07^{\mathrm{ns}}$ & $-.63^{*}$ \\
Role-Physical & $.02^{\mathrm{ns}}$ & -.21 \\
Bodily Pain & $-.24^{\mathrm{ns}}$ & -.38 \\
General Health & $-.06^{\mathrm{ns}}$ & $-.58^{*}$ \\
Vitality & $-.09^{\mathrm{ns}}$ & $-.60^{*}$ \\
Social Functioning & $.008^{\mathrm{ns}}$ & $-.71^{* *}$ \\
Role-Emotional & $-.05^{\mathrm{ns}}$ & -.37 \\
Mental Health & $-.06^{\mathrm{ns}}$ & $-.67^{* *}$ \\
\hline
\end{tabular}

BDI-II = Beck Depression Inventory, 2nd Edition. ${ }^{*} \mathrm{p}<.05 ; * * \mathrm{p}<.01$.

\section{Discussion}

FNE did not predict depression or QOL among women who maintained less weight loss after bariatric surgery. However, FNE was strongly related to depression and QOL among those who maintained greater weight loss. We propose three potential explanations.

First, patients whose social concerns are closely tied to evaluations of physical appearance and QOL may be more inclined to maintain weight loss. That is, because these individuals' level of social anxiety strongly affects their QOL, they are more motivated to engage in behavioral strategies postsurgery to maintain weight loss. We are unaware of research investigating this possibility and encourage future studies to examine weight loss outcomes in individuals whose social concerns are strongly related to their QOL even before weight loss.

A second potential explanation is related to social changes after extreme weight loss. Individuals who have lost a great deal of weight are more mobile and active in their communities, more likely to receive social attention related to drastic weight loss, and more likely to encounter social situations in which fear of negative evaluation might lead to sad mood or worsened QOL. Among patients who maintain considerable weight loss, those who still have significant worries about interpersonal evaluation may be especially prone to more psychological distress and lower quality of life. On the other hand, for people who do not lose or maintain great amounts of weight, perhaps these individuals remain generally concerned about their physical health so that social concerns play less of a role in determining QOL.

In support of this explanation, Sogg and Gorman (2008) noted that many weight loss surgery patients report receiving more positive social attention as they lose weight; however, some patients feel uncomfortable receiving compliments, and some perceive such a striking increase in positive feedback as evidence that people viewed them quite negatively before weight loss. In addition, after surgery patients often receive more romantic attention; although most patients are pleased with this change, some may feel threatened or unsure of themselves if they did not develop the social skills for dating before surgery.

A third potential explanation is that after considerable weight loss, attributions related to negative interpersonal evaluation might change. That is, prior to weight loss, many participants might have attributed negative evaluation directly to their obesity. However, if they still perceive negative evaluation after considerable weight loss, they might now attribute negative evaluation to more internalized factors rather than their weight, which may worsen mood and quality of life. In support of this 
explanation, Bocchieri, Meana, and Fisher (2002) suggested that patients may experience psychological difficulties after surgery if they tended to attribute preoperative difficulties (e.g., social rejection, low self-confidence) to their obesity. After dramatic weight loss, they are no longer able to attribute persistent life difficulties to their obesity.

\section{Limitations}

The present study is limited by a small sample size and lack of pre-surgical psychological assessment. Because the sample includes only women and is largely Caucasian, we do not know whether these results would generalize to more diverse groups. In addition, because of the correlational nature of the analyses, we cannot conclude causality. In other words, we cannot imply that FNE causes greater depression or worse QOL among patients who lose more weight after surgery; we can only state that FNE is strongly related to these other constructs. Furthermore, our study is limited by the use of telephone administration of psychosocial measures. However, although psychometric properties have not been previously reported on telephone administration of the measures in the current study, our results suggest telephone administration to be reliable and valid.

Although this is a preliminary study with clear limitations, the current research has a number of strengths. For example, this study is strengthened by a long-term follow-up period, use of specific rather than global measures of psychological functioning, examination of relationships between psychological constructs as a function of amount of weight lost, and significant results despite the small sample size. To our knowledge, this is the first study to investigate relationships between FNE and bariatric surgery outcomes.

\section{Conclusions}

Concerns about interpersonal evaluation are related to greater depressive symptoms and worse QOL among women who lose more weight after bariatric surgery. Future research is needed to replicate these findings with larger, more diverse samples and to test the validity of competing hypotheses for why FNE is associated with depression and QOL for certain individuals after surgery. Regardless of why social concerns are related to mood and QOL for certain people post-surgery, psychosocial therapy that addresses life transitions after weight loss, with particular focus on concerns about interpersonal evaluation, might improve mental health outcomes.

\section{References}

Aiken, L. S., \& West, S. G. (1991). Multiple Regression: Testing and Interpreting Interactions. Thousand Oaks: Sage.

Bocchieri, L. E., Meana, M,. \& Fisher, B. L. (2002). A review of psychosocial outcomes of surgery for morbid obesity. Journal of Psychosomatic Research, 52, 155-165. doi:10.1016/S0022-3999(01)00241-0

Beck, A.T., Steer, R. A., Ball, R., \& Ranieri, W. F. (1996). Comparison of Beck Depression Inventories-1A and II in psychiatric outpatients. Journal of Personality Assessment, 67, 588-597. doi:10.1207/s15327752jpa6703 13

Duke, D., Krishnan, M., Faith, M., \& Storch, E. A. (2006). The psychometric properties of the Brief Fear of Negative Evaluation Scale. Anxiety Disorders, 20, 807-817. doi:10.1016/j.janxdis.2005.11.002
Foster, G. D., Wadden, T. A., \& Vogt, R. A. (1997). Body image in obese women before, during, and after weight loss treatment. Health Psychology, 16, 226-229. doi:10.1037/0278-6133.16.3.226

Gilbert, N., \& Meyer, C. (2005). Fear of negative evaluation and the development of eating psychopathology: A longitudinal study among nonclinical women. International Journal of Eating Disorders, 37, 307-312. doi:10.1002/eat.20105

Grilo, C. M., Wilfley, D. E., Jones, A., Brownell, K. D., \& Rodin, J. (1994). The social self, body dissatisfaction, and binge eating in obese females. Obesity Research, 2, 24-27.

Guisado, J. A., Vaz, F. J., Alarcón, J., López-Ibor, J. J., Rubio, M. A., \& Gaite, L. (2002). Psychopathological status and interpersonal functioning following weight loss in morbidly obese patients undergoing bariatric surgery. Obesity Surgery, 12, 835-840 doi:10.1381/096089202320995664

Herpertz, S., Kielmann, R., Wolf, A. M., Langkafel, M., Senf, W., \& Hedebrand, J. (2003). Does obesity surgery improve psychosocial functioning? A systematic review. International Journal of Obesity, 27, 1300-1314. doi:10.1038/sj.ijo.0802410

Karlsson, J., Taft, C., Sjostrom, L., \& Sullivan, M. (2007). Ten-year trends in health-related quality of life after surgical and conventional treatment for severe obesity: The SOS intervention study. International Journal of Obesity, 31, 1248-1261. doi:10.1038/sj.ijo.0803573

Leary, M. R. (1983). A brief version of the Fear of Negative Evaluation Scale. Personality and Social Psychology Bulletin, 9, 371-375. doi: $10.1177 / 0146167283093007$

Lundgren, J. D., Anderson, D. A., \& Thompson, J. K. (2004). Fear of negative appearance evaluation: Development and evaluation of a new construct for risk factor work in the field of eating disorders. Eating Behaviors, 5, 75-84. doi:10.1016/S1471-0153(03)00055-2

Mathus-Vliegen, E. M. H. (2007). Long-term health and psychosocial outcomes from surgically induced weight loss: Results obtained in patients not attending protocolled follow-up visits. International Journal of Obesity, 31, 299-307. doi:10.1038/sj.ijo.0803404

McClintock, J. M., \& Evans, I. M. (2001). The underlying psychopathology of eating disorders and social phobia: A structural equation analysis. Eating Behaviors, 2, 247-261. doi:10.1016/S1471-0153(01)00032-0

Myers, V. H., Adams, C. E., Barbera, B., L., \& Brantley, P. J. (in press). Medical and psychosocial outcomes of laparoscopic Roux-en-Y gastric bypass: Cross-sectional findings at 4-year follow-up. Obesity Surgery, in press. doi:10.1007/s11695-010-0324-7

Petroni, M. L., Villanova, N., Avagnina, S., Fusco, M. A., Fatati, G., \& Compare, A., et al. (2007). Psychological distress in morbid obesity in relation to weight history. Obesity Surgery, 17, 391-399. doi:10.1007/s11695-007-9069-3

Rodebaugh, T. L., Woods, C. M., Thissen, D. M., Heimberg, R. G., Chambless, D. L., \& Rapee, R. M. (2004). More information from fewer questions: The factor structure and item properties of the Original and Brief Fear of Negative Evaluation Scale. Psychological Assessment, 16, 169-181. doi:10.1037/1040-3590.16.2.169

Sarwer, D. B., Fabricatore, A. N., Eisenberg, M. H., Sywulak, L. A., \& Wadden, T. A. (2008). Self-reported stigmatization among candidates for bariatric surgery. Obesity, 16, S75-S79. doi: $10.1038 /$ oby. 2008.450

Sogg, S., \& Gorman, M. J. (2008). Interpersonal changes and challenges after weight-loss surgery. Primary Psychiatry, 15, 61-66.

Tabachnick, B. G., \& Fidell, L. S. (2007). Using Multivariate Statistics (5th ed.). Boston: Pearson.

Ware, J. E. (1993). SF-36 Health survey: Manual and interpretation guide. Boston, MA: The Health Institute, New England Medical Center.

Waters, G. S., Pories, W. J., Swanson, M. S., Meelheim, H. D., Flickinger, E. G., \& May, H. J. (1991). Long-term studies of mental health after the Greenville gastric bypass operation for morbid obesity. American Journal of Surgery, 161, 154-157. doi:10.1016/0002-9610(91)90377-P

Watson, D., \& Friend, R. (1969). Measurement of social-evaluative anxiety. Journal of Consulting and Clinical Psychology, 33, 448-457. doi:10.1037/h0027806 